\title{
Magnetic and Mössbauer characterization of the magnetic properties of single-crystalline sub-micron sized $\mathrm{Bi}_{2} \mathrm{Fe}_{4} \mathrm{O}_{9}$ cubes
}

Georgia C. Papaefthymiou ${ }^{1,{ }^{*}}$, Arthur J. Viescas ${ }^{1}$, Jean-Marie Le Breton ${ }^{2,{ }^{*}}$, Hubert Chiron $^{2}$, Jean Juraszek ${ }^{2}$, Tae-Jin Park ${ }^{3, \#}$ and Stanislaus S. Wong ${ }^{3,4}$

${ }^{I}$ Department of Physics, Villanova University, Villanova, PA 19085, USA

${ }^{2}$ Groupe de Physique des Matériaux, UMR 6634 CNRS, Université et INSA de Rouen, Saint

Etienne du Rouvray, Cedex, France

${ }^{3}$ Department of Chemistry, State University of New York at Stony Brook, Stony Brook, NY 11794 3400 USA

${ }^{4}$ Condensed Matter Physics and Materials Sciences Department, Brookhaven National Laboratory, Building 480, Upton, NY 11973, USA

${ }^{\#}$ Current Address: Korea Atomic Energy Research Institute (KAERI), 989-111 Daedoek-daero, Yuseong, Daejeon, Korea 305-353

Magnetic and Mössbauer characterization of single crystalline, sub-micron sized $\mathrm{Bi}_{2} \mathrm{Fe}_{4} \mathrm{O}_{9}$ cubes has been performed using SQUID magnetometry and transmission Mössbauer spectroscopy in the temperature range of $4.2 \mathrm{~K} \leq \mathrm{T} \leq 300 \mathrm{~K}$. A broad magnetic phase transition from the paramagnetic to the anti-ferromagnetic state is observed below $250 \mathrm{~K}$, with the Mössbauer spectra exhibiting a superposition of magnetic, collapsed and quadrupolar spectra in the transition region of $200 \mathrm{~K}<\mathrm{T}<245$ K. Room temperature Mössbauer spectra obtained in transmission geometry are identical to those recorded in back-scattering geometry via conversion electron Mössbauer spectroscopy, indicating the absence of strain at the surface. A small hysteresis loop is observed in SQUID measurements at $5 \mathrm{~K}$, attributable to the presence of weakferromagnetism arising from the canting of $\mathrm{Fe}^{3+}$ ion sublattices in the antiferromagnetic matrix. 
*Author to whom correspondence should be addressed. Electronic mail: georgia.papaefthymioudavis@ villanova.edu *Author to whom correspondence should be addressed. Electronic mail: jean-marie.lebreton@univ-rouen.fr

\section{INTRODUCTION}

The family of metal oxides of the form $\mathrm{Bi}_{2} \mathrm{M}_{4} \mathrm{O}_{9}(\mathrm{M}=\mathrm{Fe}, \mathrm{Mn}, \mathrm{Al}$, or $\mathrm{Ga})$, and their solid solutions, possesses a pseudo planar structure in which bismuth oxide planes sandwich metal oxide regions that contain tetrahedral and octahedral coordination sites and exhibit rich magnetic properties [1, 2]. For $\mathrm{Bi}_{2} \mathrm{Fe}_{4} \mathrm{O}_{9}(\mathrm{M}=\mathrm{Fe})$, the presence of iron affords detailed micro-magnetic characterization by the use of two complementary techniques, namely SQUID magnetometry and Mössbauer spectroscopy, with vastly different characteristic measurement time windows. While such an approach has been employed in the study of this system, and its solid solutions with Al and $\mathrm{Ga}$ in bulk form [1,2], little is known of their magnetic properties in the micrometer size regime. In particular, very few reports associated with micro- or nano-structural studies of $\mathrm{Bi}_{2} \mathrm{Fe}_{4} \mathrm{O}_{9}$ have appeared in the literature $[3,4]$. Possible novel behavior at the micron scale could make such systems promising candidates for a variety of applications. Advanced synthesis techniques, which promote particle growth along a particular direction with tailored particle shape and exposed crystallographic faces at the particle's surface, promise to produce new nanomaterials for gas sensors and other future device applications [5]. In addition, remarkable multiferroic effects have been reported in polycrystalline $\mathrm{Bi}_{2} \mathrm{Fe}_{4} \mathrm{O}_{9}$, which presents both antiferromagnetic order and ferroelectric hysteresis [6]

Herewith, we report on the magnetic and electronic properties of single-crystalline submicron sized $\mathrm{Bi}_{2} \mathrm{Fe}_{4} \mathrm{O}_{9}$ cubes, the synthesis of which and preliminary magnetic characterization have 
already been previously reported [3]. A combination of transmission and conversion electron Mössbauer spectroscopic measurements was employed to further characterize iron surface states. Interest in surface states derives from the fact that $\mathrm{Bi}_{2} \mathrm{Fe}_{4} \mathrm{O}_{9}$ exhibits catalytic activity for the oxidation of $\mathrm{NH}_{3}$ to $\mathrm{NO}$ and may lead to the production of iron-based catalysts in the future to eventually replace current, expensive Pt-, Rh-, and Pd-based catalyst systems [4, 7].

\section{MATERIALS}

The detailed synthesis procedures, X-ray diffraction (XRD), as well as Transmission and Scanning Electron Microscopy (TEM, SEM) data on these mono-crystalline $\mathrm{Bi}_{2} \mathrm{Fe}_{4} \mathrm{O}_{9}$ submicron-cubes were reported elsewhere [3]. A facile, large scale solid-state reaction employing a molten salt technique in the presence of nonionic surfactants was used. In general, stoichiometric amounts of $\mathrm{Bi}_{2} \mathrm{O}_{3}, \mathrm{Fe}_{2} \mathrm{O}_{3}$, Igepal or NP-9 surfactant, and $\mathrm{NaCl}$ were mixed to generate sub-micron sized cubes of $\mathrm{Bi}_{2} \mathrm{Fe}_{4} \mathrm{O}_{9}$, the crystal structure of which was confirmed by XRD measurements [3]. Figure 1-A shows SEM images revealing the morphologies of asprepared $\mathrm{Bi}_{2} \mathrm{Fe}_{4} \mathrm{O}_{9}$. It can be seen that the $\mathrm{Bi}_{2} \mathrm{Fe}_{4} \mathrm{O}_{9}$ product consists mainly of cubic structures. Their mean edge-length was determined to be $386 \mathrm{~nm}$ with a standard deviation of $\pm 147 \mathrm{~nm}$, though particles were formed with an overall size range from $~ 166$ to $833 \mathrm{~nm}$. It is observed that the faces of the $\mathrm{Bi}_{2} \mathrm{Fe}_{4} \mathrm{O}_{9}$ structures are essentially flat, though some of the corners and edges of these structures are slightly truncated. Figure 1-B shows a representative individual $\mathrm{Bi}_{2} \mathrm{Fe}_{4} \mathrm{O}_{9}$ structure with truncated edges which could be schematically described by the illustration shown in the inset. By varying the molar ratios of the starting materials, a variety of structural motifs could be obtained from cubic to elongated parallelepiped particles [3]. 


\section{EXPERIMENTAL}

As synthesized, polycrystalline samples were studied. Magnetization measurements were performed using a Quantum Design MPMS SQUID magnetometer. Zero-Field-Cooled/FieldCooled (ZFC/FC) magnetization curves were obtained under an applied field of $4 \mathrm{kA} \cdot \mathrm{m}^{-1}$ (50 Oe). Isothermal, magnetization vs applied field $(\mathrm{M}(\mathrm{H}))$ curves were measured between 5 and 300 K, with a maximum applied field of either $1600 \mathrm{kA} \cdot \mathrm{m}^{-1}$ or $4000 \mathrm{kA} \cdot \mathrm{m}^{-1}$.

Transmission Mössbauer Spectroscopy (TMS) studies were carried out both at room temperature and at low temperatures using a conventional, constant acceleration Mössbauer spectrometer. Low temperatures were achieved with a Janis Research Co. Supervaritemp cryostat. Conversion Electron Mössbauer Spectroscopy (CEMS) was performed at room temperature in reflection mode using a home-made spectrometer operating in constant acceleration mode from $-10 \mathrm{~mm} / \mathrm{s}$ to $+10 \mathrm{~mm} / \mathrm{s}$ [8]. The source was a $30-\mathrm{mCi}^{-57} \mathrm{Co}$ radioactive source in Rh matrix, maintained at room temperature. The experimental TMS data were leastsquare fitted to theoretical spectra using the software package WMOSS by SEE Co (Edina, Minnesota) and assuming Lorentzian absorption lines. The CEMS data were fitted using the MOSFIT program. Isomer shifts are reported relative to metallic iron at room temperature. Estimated errors for the hyperfine parameters originate from the statistical errors $\sigma$ given by the fitting program and taking $3 \sigma$ to be the error associated with each measured parameter. 


\section{RESULTS}

\section{A. Magnetization measurements}

The $\mathrm{M}(\mathrm{H})$ measurements of the $\mathrm{Bi}_{2} \mathrm{Fe}_{4} \mathrm{O}_{9}$ powder sample were obtained at temperatures ranging from 5 to $300 \mathrm{~K}$. All the curves look similar, revealing an antiferromagnetic behaviour in the whole temperature range, in agreement with previous literature [3]. At $5 \mathrm{~K}$, the presence of a weak ferromagnetic component is detected as revealed by the hysteresis loops shown in figure 2 . Such a behavior has previously been reported for $\mathrm{Bi}_{2} \mathrm{Fe}_{4} \mathrm{O}_{9}$ powders and sub-micrometer sized particles, at 5K $[9,10]$.

The slope of the $\mathrm{M}(\mathrm{H})$ curve at high field is proportional to the susceptibility $\chi_{\mathrm{m}}$ of the antiferromagnetic contribution. The temperature dependence of $\chi_{\mathrm{m}}$ is shown in figure 3 . The values reported in the figure are deduced from all the $\mathrm{M}(\mathrm{H})$ measurements performed on the sample in the whole range of temperature. This curve is comparable with the temperature dependence of the magnetic susceptibility measured under an applied field of about $1000 \mathrm{Oe}$, of the same material [3] and, with the ZFC curves of submicron or polycrystalline $\mathrm{Bi}_{2} \mathrm{Fe}_{4} \mathrm{O}_{9}$ samples prepared by other groups $[6,9]$. It is worth mentioning that the curve in figure 3 is similar to the temperature dependence of magnetization under a $1 \mathrm{~T}$ field reported for $200 \mathrm{~nm}$ sized powders [11], in reasonable agreement with the size of our sub-micron particles. This reflects the antiferromagnetic behaviour of the powders. The Néel temperature, corresponding to the maximum of the $\chi_{\mathrm{m}}(\mathrm{T})$ curve, is around $250 \mathrm{~K}$, in good agreement with previous results [3]. Two features of the susceptibility vs. temperature curve of figure 3 are noteworthy; (a) the broadness of the transition around $\mathrm{T}_{\mathrm{N}}=250 \mathrm{~K}$ and $(\mathrm{b})$ the sharp rise of the susceptibility below $10 \mathrm{~K}$. These features are further discussed below in conjunction with the Mössbauer results. 
The weak ferromagnetism in $\mathrm{Bi}_{2} \mathrm{Fe}_{4} \mathrm{O}_{9}$ powders at $5 \mathrm{~K}$ and rise of the susceptibility at low temperatures cannot could be attributed to particle size effects [12], due to uncompensated spins on the two Fe sublattices [13], because the particles are too large to exhibit such finite size effects. It is rather attributed spin-sublattice canting in the antiferromagnetic lattice of $\mathrm{Bi}_{2} \mathrm{Fe}_{4} \mathrm{O}_{9}$ [10] and the possible presence of structural defects, as further elaborated below.

\section{B. Transmission Mössbauer Spectroscopy}

Mössbauer data in the temperature range from $4.2 \mathrm{~K}$ to $\mathrm{RT}$ are shown in figure 4 , with associated Mössbauer parameters obtained from least-square fits of the experimental data to a superposition of spectral signatures presented in table 1. With decreasing temperature, the sample passes from the paramagnetic to a magnetically ordered phase at or below $250 \mathrm{~K}$. In the bulk, $\mathrm{Bi}_{2} \mathrm{Fe}_{4} \mathrm{O}_{9}$ has been reported to enter into an antiferromagnetically ordered phase at $\mathrm{T}_{\mathrm{N}}=$ $263 \mathrm{~K}$ [14], although somewhat lower transition temperatures, ranging from $237 \mathrm{~K}$ to $263 \mathrm{~K}$, have also been reported $[1,15-20]$. In polycrystalline $\mathrm{Bi}_{2} \mathrm{Fe}_{4} \mathrm{O}_{9}$ particles, $\mathrm{T}_{\mathrm{N}}$ shifts to $229 \mathrm{~K}$, as the grain size decreases to $60 \mathrm{~nm}[11]$.

The fractional areas of the two contributions that are related to the tetrahedral and octahedral Fe sites are the same in the whole range of temperature, within the degree of precision of the measurements. However, a slight disagreement is observed at low temperatures, where f-factors are expected to be similar. This could be related to the presence of a small amount of iron vacancies at the octahedral site or the possible inadvertent substitution of $\mathrm{Fe}^{3+}$ with some impurity ions.

At room temperature, the spectra consist of the superposition of two quadrupole doublets of about equal intensity with an isomer shift $\delta_{1}=0.24 \mathrm{~mm} / \mathrm{s}$ and a quadrupole splitting $\Delta_{1}=0.94$ 
$\mathrm{mm} / \mathrm{s}$ associated with the tetrahedral site (red line), and $\delta_{2}=0.36 \mathrm{~mm} / \mathrm{s}, \Delta_{2}=0.38 \mathrm{~mm} / \mathrm{s}$ associated with the octahedral site (blue line). The $250 \mathrm{~K}$ spectrum is similar to the RT spectrum, except for slightly broadened absorption lines.

Considering the overall Mössbauer spectral temperature profile of figure 4, four different regions can be distinguished as we move from low to high temperatures, as indicated on Table 1 . Focusing first on the magnetic sub-structure of the spectra, region I consists of the low temperature behavior, at $4.2 \mathrm{~K}$ and $135 \mathrm{~K}$ (figure 4 , h and g, respectively). At $4.2 \mathrm{~K}$ two sharp magnetic subsites are clearly resolved associated with the octahedral (dark blue line) and tetrahedral (dark red line) iron sub-sites. At $135 \mathrm{~K}$ an additional broadened weak spectral feature (green line) had to be added to obtain good least-square fits to the data. In region II, at intermediate temperatures $200 \mathrm{~K}$ and $220 \mathrm{~K}$, the spectra are magnetic, but due to the well-known temperature dependence of the hyperfine magnetic fields shown in figure 5, octahedral and tetrahedral site signatures are no longer clearly resolved, except by spectral fitting deconvolution. The broad background spectral feature first observed at $135 \mathrm{~K}$ is intensified (green line). The origin of this feature may be associated with a spin-reorientation transition previously reported for $\mathrm{Bi}_{2} \mathrm{Fe}_{4} \mathrm{O}_{9}$ to occur around $200 \mathrm{~K}$ [1], or possibly the presence of particles of low crystallinity. In region III, spectra were collected at $240 \mathrm{~K}$ and $245 \mathrm{~K}$. In this region the magnetic spectra of the tetrahedral and octahedral subsites could no longer be de-convoluted (green line, figure 4, d and c). In addition, a collapsed broad absorption background (violet line) and strong quadrupolar components are observed (red and blue lines, figure 4, c and d). Region III is associated with the magnetic phase transition from the paramagnetic to the anti-ferromagnetic state. The broadening of the magnetic spectra and collapsed spectral feature are associated with spin fluctuations around the Néel temperature as the sample passes from the paramagnetic, quadrupolar, to the 
antiferromagnetic, magnetically split, state. The coexistence of quadrupolar, collapsed and broadened magnetically split spectral features in this regime are consistent with the observation of a broad magnetic phase transition in figure 3. As the magnetic phase transition is mediated by superexchange interactions between $\mathrm{Fe}$ ions via $\mathrm{O}$ atoms, the presence of oxygen nonstoichiometry, lack of crystallinity, iron vacancies or iron substitutions with non-magnetic ions $[1,2]$ may contribute to the breadth of the transition. The latter will also result in spin noncompensation that may give rise to the sharp increase of the magnetic susceptibility at low temperatures observed in figure 3. Region IV, at $250 \mathrm{~K}$ and $293 \mathrm{~K}$, consists of the paramagnetic regime above the Néel temperature, where a superposition of two quadrupole doublets is observed (red and blue lines, figure 4, b and a). The Néel temperature estimated from the TMS analysis, as well as the breadth of the transition, is also in good agreement with zero-field cooled magnetization measurements of the same material [3] and of submicron or polycrystalline $\mathrm{Bi}_{2} \mathrm{Fe}_{4} \mathrm{O}_{9}$ samples prepared by other groups $[6,9,11]$.

\section{Conversion Electron Mössbauer Spectroscopy}

Due to the interesting catalytic properties of $\mathrm{Bi}_{2} \mathrm{Fe}_{4} \mathrm{O}_{9}$, the surface iron states of these submicron cubes were also studied by using CEMS in reflection geometry. Figure 6 compares the TMS and CEMS spectra at room temperature. Identical spectra are observed in either geometry, satisfactorily fitted with the same Mössbauer parameters (Table 2). Coordination distortions at the surface, due to the sudden interruption of crystallographic and magnetic order, are generally expected to produce surface strain with larger electric field gradients at the surface resulting in larger quadrupole splittings compared to interior iron sites. The lack of detected distortions in the CEMS study would thus indicate the absence of surface strain on the analyzed thickness (about 
$50 \mathrm{~nm}$ ). This may be the result of epitaxial-like crystal growth along a preferred direction. XRD structure characterization previously reported [3] indicated that the observed diffraction peaks could be indexed to the orthorhombic structure of $\mathrm{Bi}_{2} \mathrm{Fe}_{4} \mathrm{O}_{9}$ (space group: Pbam) with lattice constants of $a=7.965 \AA, b=8.440 \AA$, and $c=5.994 \AA$. In addition, the relative intensities of the reflection of the (220) signal to that of the other peaks were found to be stronger than those of the corresponding bulk materials, an observation which can be ascribed to the preferential formation of the (220) orientation during the synthesis process. An alternate explanation may lie in the fact that the presence of surfactant on the surface adds additional stress that counteracts surface strain. Thus, our CEMS data confirms the results deduced from the XRD studies.

\section{SUMMARY AND CONCLUSION}

The SQUID measurements and Mössbauer analyses on the sub-micron sized $\mathrm{Bi}_{2} \mathrm{Fe}_{4} \mathrm{O}_{9}$ cubes show a broad paramagnetic-to-antiferromagnetic phase transition to occur over the temperature range of about $240-250 \mathrm{~K}$. In the temperature range $200 \mathrm{~K}<\mathrm{T}<245 \mathrm{~K}$ the spectra exhibit a superposition of spectral features that can be attributed to dynamic spin processes occurring around $\mathrm{T}_{\mathrm{N}}$ and a spin reorientation transition previously reported to occur at $200 \mathrm{~K}$. The small paramagnetic contributions persisting down to $200 \mathrm{~K}$ may be associated with defects in the crystallographic structure. Their contribution to the spectral area is however too close to the associated error of measurement to be particularly meaningful. More importantly, the fact that surface sites give identical Mössbauer signatures as interior sites indicates the absence of strain at the surface. This observation confirms that crystal growth occurs along a preferential orientation within the sub-micron sized cubes, as previously reported, based on XRD 
measurements. The presence of a weak ferromagnetic component at $5 \mathrm{~K}$ could be related to canting of the antiferromagnetic sublattices, as previously reported for $\mathrm{Bi}_{2} \mathrm{Fe}_{4} \mathrm{O}_{9}$ powders.

\section{ACKNOWLEDGEMENTS}

GCP thanks the National Science Foundation for support at Villanova University. The synthesis research (including support for SSW) reported herein was supported by the U.S. Department of Energy, Basic Energy Sciences, Materials Sciences and Engineering Division. 


\section{REFERENCES}

1. D. M. Giaquinta, G. C. Papaefthymiou, W. M. Davis and H.-C. Zur Loye, J. Sol. State Chem. 99, $120(1992)$

2. D. M. Giaquinta, G. C. Papaefthymiou, W. M. Davis and H.-C. Zur Loye, J. Sol. State Chem. 114, 199 (1995)

3. T.-J. Park, G. C. Papaefthymiou, A. R. Moodenbaugh, Y. Mao and S. S. Wong, J. Mater. Chem. 15, 2099 (2005); and references there in

4. Y. Xiong, M. Wu, Z. Peng, N. Jiang, Q. Chen, Chem. Lett. 33, 502 (2004)

5. W. P. Lim, Z. Zhang, H. Y. Low, W. S. Chin, Angew. Chem. Int. Ed. 43, 5685 (2004)

6. A. K. Singh, S. D. Kaushik, B. Kumar, P. K. Mishra, A. Venimadhav, V. Siruguri, S. Patnaik, App. Phys. Lett. 92, 132910 (2008)

7. N. I. Zakharchenko, Kinet. Catal. 43, 95 (2002)

8. J. Juraszek, O. Zivotsky, H. Chiron, C. Vaudolon, J. Teillet, Rev. Sci. Instrum. 80, 043905 (2009)

9. J.T. Han, Y.H. Huang, R.J. Jia, G.C. Shan, R.Q. Guo, W. Huang, J. Cryst. Growth 294, 469 (2006)

10. G. Alvarez, J. Contrerars, A. Conde-Gallardo, H. Montiel and R. Zamorano, J. Mag. Mag. Mater. 348, 17-21 (2013)

11. Z.M. Tian, S.L. Yuan, X.L. Wang, X.F. Zheng, S.Y. Yin, C.H. Wang, L. Liu, J. Appl. Phys. $106,103912(2009)$

12. T.-J. Park, G.C. Papaefthymiou, A.J. Viescas, A.R. Moodenbaugh, S.S. Wong, Nano Lett. 7, $766(2007)$

13. L. Néel, Compt. Rend. Acad. Sci. 252, 4075 (1961)

14. N. Shamir, E. Gurewitz, Acta Crystallogr. A 34, 662 (1978)

15. V. A. Bokov, G. V. Novikov, V. A. Trukhtanov, S. I. Yushchuk, Sov. Phys. Solid State 11, $2324(1970)$

16. D. Groult, M. Hervieu, N. Nguyen, B. Raveau, J. Solid State Chem. 76, 248 (1988)

17. A.G. Tutov, I.E. Myl'nikova, N.N. Parfenova, V.A. Bokov, S.A. Kizhaev, Sov. Phys. Solid State 6, 963 (1964)

18. Y.A. Park, K.M. Song, K.D. Lee, C.J. Won, N. Hur, Appl. Phys. Lett. 96, 092506 (2010)

19. Y. Du, Z.X. Cheng, S.X. Dou, X.L. Wang, Mat. Letters 64, 2251 (2010) 
20. M.N. Iliev, A.P. Litvinchuk, V.G. Hadjiev, M.M. Gospodinov, V. Skumryev, E. Ressouche, Phys. Rev. B 81, 024302 (2010) 
Table 1. Fitted Mössbauer hyperfine parameters corresponding to the transmission spectra of the $\mathrm{Bi}_{2} \mathrm{Fe}_{4} \mathrm{O}_{9}$ sub-micron cubes at the indicated temperatures.

\begin{tabular}{|c|c|c|c|c|c|c|c|}
\hline $\begin{array}{c}T \\
(K)\end{array}$ & Site & $\begin{array}{c}\Gamma \\
(\mathrm{mm} / \mathrm{s}) \\
\pm 0.02 \mathrm{~mm} / \mathrm{s} \\
\end{array}$ & $\begin{array}{c}\delta \\
(\mathrm{mm} / \mathrm{s}) \\
\pm 0.02 \mathrm{~mm} / \mathrm{s} \\
\end{array}$ & $\begin{array}{c}\Delta \text { or } \varepsilon \\
(\mathrm{mm} / \mathrm{s}) \\
\pm 0.02 \mathrm{~mm} / \mathrm{s} \\
\end{array}$ & $\begin{aligned} & B_{\mathrm{hf}} \\
&(\mathrm{T}) \\
& \pm 0.2 \mathrm{~T} \\
&\end{aligned}$ & $\begin{array}{c}\text { Fractional } \\
\text { Area }(\%) \\
\pm 2 \%\end{array}$ & Phase \\
\hline \multicolumn{8}{|c|}{ Region IV } \\
\hline \multirow[t]{2}{*}{293} & Tetra. & 0.30 & 0.24 & 0.94 & - & 51 & paramagnetic \\
\hline & Oct. & 0.29 & 0.36 & 0.38 & - & 49 & paramagnetic \\
\hline \multirow[t]{2}{*}{250} & Tetra. & 0.34 & 0.28 & 0.98 & - & 50 & paramagnetic \\
\hline & Oct. & 0.35 & 0.40 & 0.39 & - & 50 & paramagnetic \\
\hline \multicolumn{8}{|c|}{ Region III } \\
\hline \multirow[t]{4}{*}{245} & Tetra. & 0.30 & 0.30 & 0.98 & - & 10 & paramagnetic \\
\hline & Oct. & 0.30 & 0.40 & 0.42 & - & 12 & paramagnetic \\
\hline & Both & 0.22 & 0.31 & 0.16 & 18 & 38 & magnetic \\
\hline & Both & 2.14 & 0.25 & - & - & 40 & collapsed \\
\hline \multirow[t]{4}{*}{240} & Tetra. & 0.29 & 0.27 & 0.84 & - & 7 & paramagnetic \\
\hline & Oct. & 0.29 & 0.38 & 0.37 & - & 6 & paramagnetic \\
\hline & Both & 0.22 & 0.34 & 0.22 & 22.4 & 53 & magnetic \\
\hline & Both & 3.4 & 0.29 & - & - & 34 & collapsed \\
\hline \multicolumn{8}{|c|}{ Region II } \\
\hline \multirow[t]{5}{*}{220} & Tetra. & 0.34 & 0.34 & 0.80 & - & 4 & super-paramagnetic \\
\hline & Oct. & 0.35 & 0.40 & 0.41 & - & 4 & super-paramagnetic \\
\hline & Tetra. & 0.22 & 0.27 & 0.25 & 31.5 & 29 & magnetic \\
\hline & Oct. & 0.22 & 0.44 & 0.23 & 31.7 & 29 & magnetic \\
\hline & Both & 0.54 & 0.375 & 0.15 & 26.8 & 34 & $\begin{array}{c}\text { intermediate } \\
\text { relaxation }\end{array}$ \\
\hline \multirow[t]{5}{*}{200} & Tetra. & 0.34 & 0.29 & 0.95 & - & 3 & super-paramagnetic \\
\hline & Oct. & 0.35 & 0.41 & 0.39 & - & 3 & super-paramagnetic \\
\hline & Tetra. & 0.22 & 0.29 & 0.275 & 35.4 & 32 & magnetic \\
\hline & Oct. & 0.22 & 0.41 & 0.15 & 36.5 & 32 & magnetic \\
\hline & Both & 0.45 & 0.39 & 0.15 & 33.0 & 30 & $\begin{array}{l}\text { intermediate } \\
\text { relaxation }\end{array}$ \\
\hline \multicolumn{8}{|c|}{ Region I } \\
\hline \multirow[t]{3}{*}{135} & Tetra. & 0.20 & 0.33 & 0.12 & 42.3 & 46 & magnetic \\
\hline & Oct. & 0.20 & 0.47 & 0.19 & 44.8 & 46 & magnetic \\
\hline & Both & 0.28 & 0.30 & 0.04 & 39.0 & 8 & $\begin{array}{c}\text { Intermediate } \\
\text { relaxation }\end{array}$ \\
\hline \multirow[t]{2}{*}{4.2} & Tetra. & 0.22 & 0.35 & 0.05 & 46.8 & 52 & magnetic \\
\hline & Oct. & 0.22 & 0.48 & 0.17 & 50.7 & 48 & magnetic \\
\hline
\end{tabular}


Table 2. Fitted Mössbauer hyperfine parameters corresponding to the CEMS and TMS spectra of the $\mathrm{Bi}_{2} \mathrm{Fe}_{4} \mathrm{O}_{9}$ sub-micron cubes at room temperature.

\begin{tabular}{|c|c|c|c|c|c|}
\hline Geometry & Site & $\begin{array}{c}\Gamma \\
(\mathrm{mm} / \mathrm{s}) \\
\pm 0.02 \mathrm{~mm} / \mathrm{s} \\
\end{array}$ & $\begin{array}{c}\delta \\
(\mathrm{mm} / \mathrm{s}) \\
\pm 0.02 \mathrm{~mm} / \mathrm{s} \\
\end{array}$ & $\begin{array}{c}\Delta \\
(\mathrm{mm} / \mathrm{s}) \\
\pm 0.02 \mathrm{~mm} / \mathrm{s} \\
\end{array}$ & $\begin{array}{c}\text { Fractional } \\
\text { Area (\%) } \\
\pm 2 \% \\
\end{array}$ \\
\hline \multirow[t]{2}{*}{ CEMS } & Tetra. & 0.34 & 0.24 & 0.93 & 52 \\
\hline & Oct. & 0.34 & 0.36 & 0.38 & 48 \\
\hline \multirow[t]{2}{*}{ TMS } & Tetra. & 0.36 & 0.25 & 1.02 & 51 \\
\hline & Oct. & 0.36 & 0.38 & 0.39 & 49 \\
\hline
\end{tabular}




\section{Figure Captions}

FIG. 1. SEM images of a collection of $\mathrm{Bi}_{2} \mathrm{Fe}_{4} \mathrm{O}_{9}$ cubes prepared using a molten salt method (A), and a typical individual cube (B). The inset illustrates a schematic of the facets of an individual cube (from [3]).

FIG. 2. $\mathrm{M}(\mathrm{H})$ curve of the $\mathrm{Bi}_{2} \mathrm{Fe}_{4} \mathrm{O}_{9}$ sample at $5 \mathrm{~K}$.

FIG. 3. Temperature dependence of the mass susceptibility of the antiferromagnetic contribution, deduced from the $\mathrm{M}(\mathrm{H})$ curves of the $\mathrm{Bi}_{2} \mathrm{Fe}_{4} \mathrm{O}_{9}$ sample. The dashed line is a guide for the eye.

FIG. 4. Transmission Mössbauer spectra of $\mathrm{Bi}_{2} \mathrm{Fe}_{4} \mathrm{O}_{9}$ sub-micron cubes from room to liquid helium temperature. The solid lines through the experimental points are least square fits of the experimental data to spectral convolutions of superimposed iron sub-sites indicated by solid lines above the experimental points; ( $\mathrm{a}$ and $\mathrm{b}$ ) quadrupole doublet due to octahedral iron sites (red) and tetrahedral iron sites (blue); (c) and (d) quadrupole doublets - octahedral (red) and tetrahedral (blue), magnetic (green), collapsed (violet); (e) and (f) quadrupolar (red and blue), magnetic-octahedral (dark blue), tetrahedral (dark red), unresolved magnetic (green); (g) and (h) magnetic- octahedral (dark blue), tetrahedral (dark red) unresolved magnetic (green).

FIG. 5. Temperature dependence of the magnetic hyperfine field of the two magnetic components used to fit the Mössbauer spectrum of the $\mathrm{Bi}_{2} \mathrm{Fe}_{4} \mathrm{O}_{9}$ cubes. The open circles and open triangles 
correspond to the octahedral and tetrahedral sites, respectively. The solid lines are spline curves through the experimental points.

FIG. 6. TMS (a) and CEMS (b) spectra of $\mathrm{Bi}_{2} \mathrm{Fe}_{4} \mathrm{O}_{9}$ samples at room temperature. 


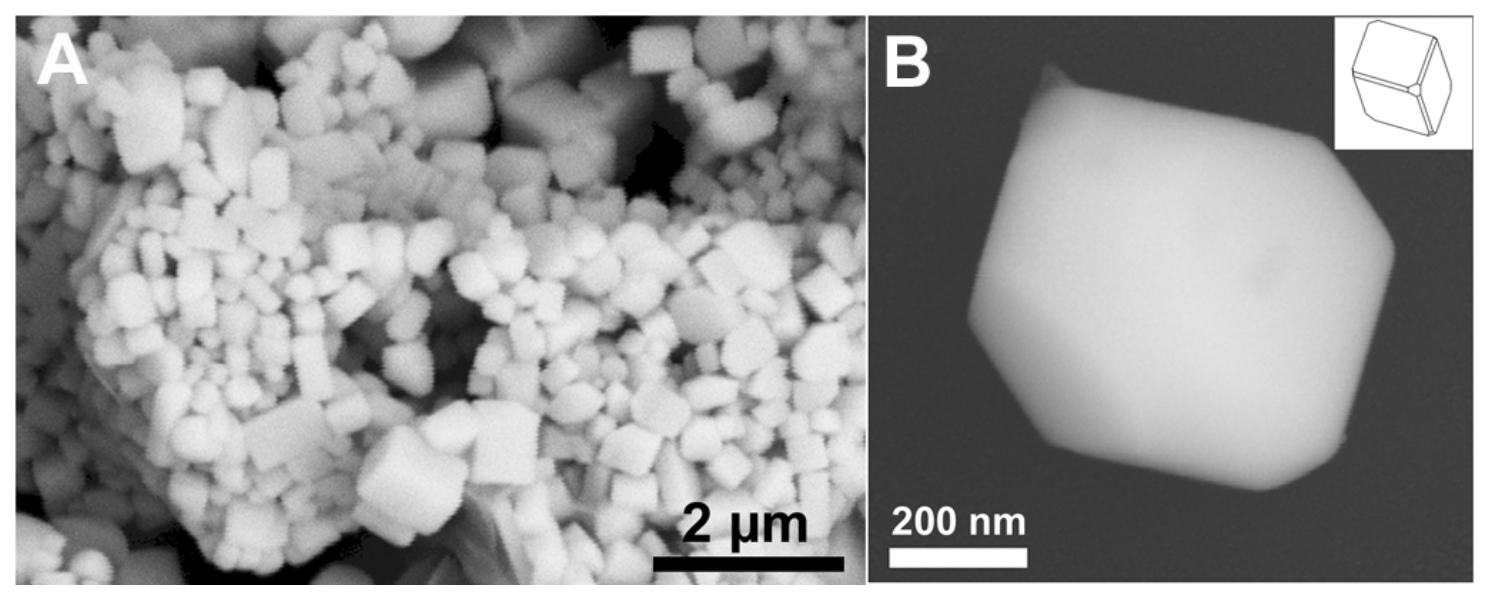

FIG. 1. 


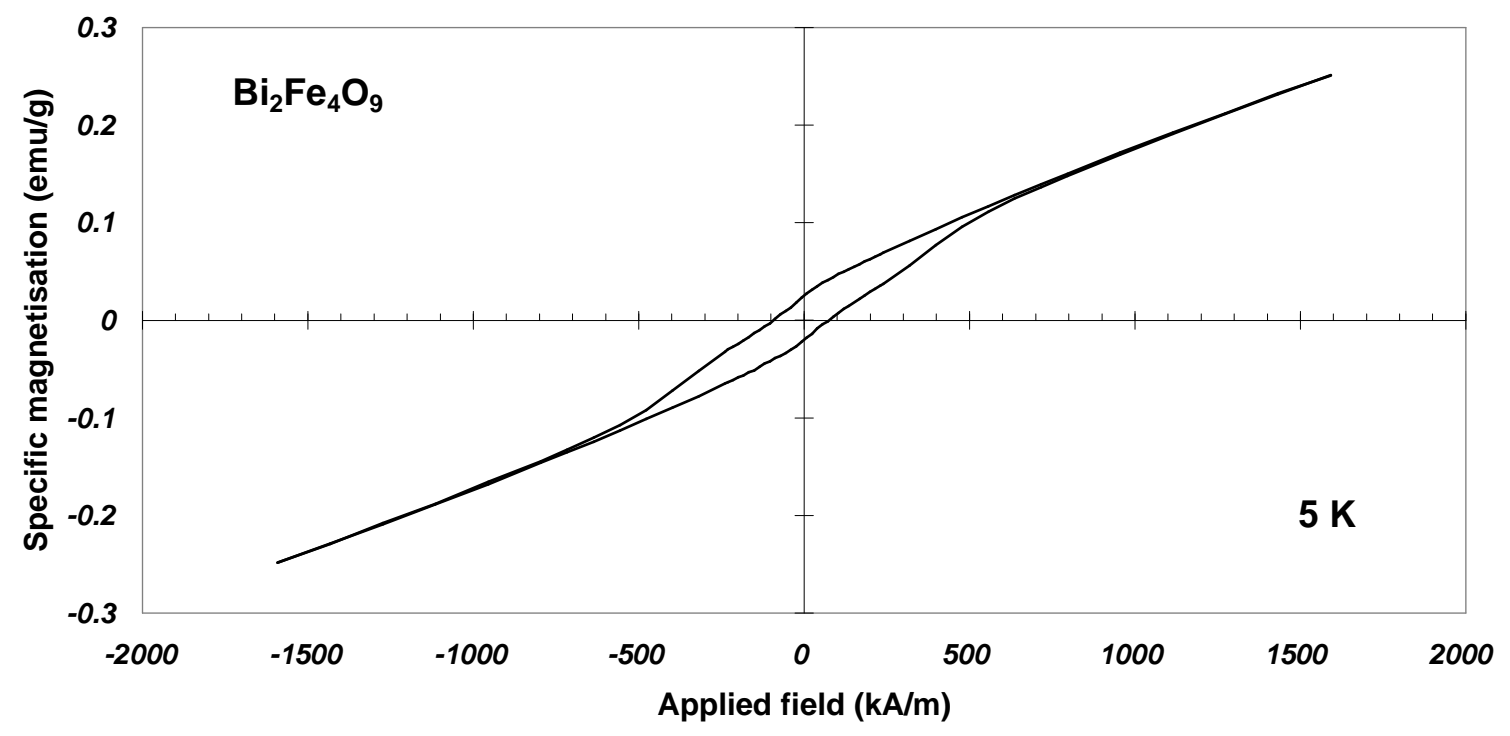

FIG. 2. 


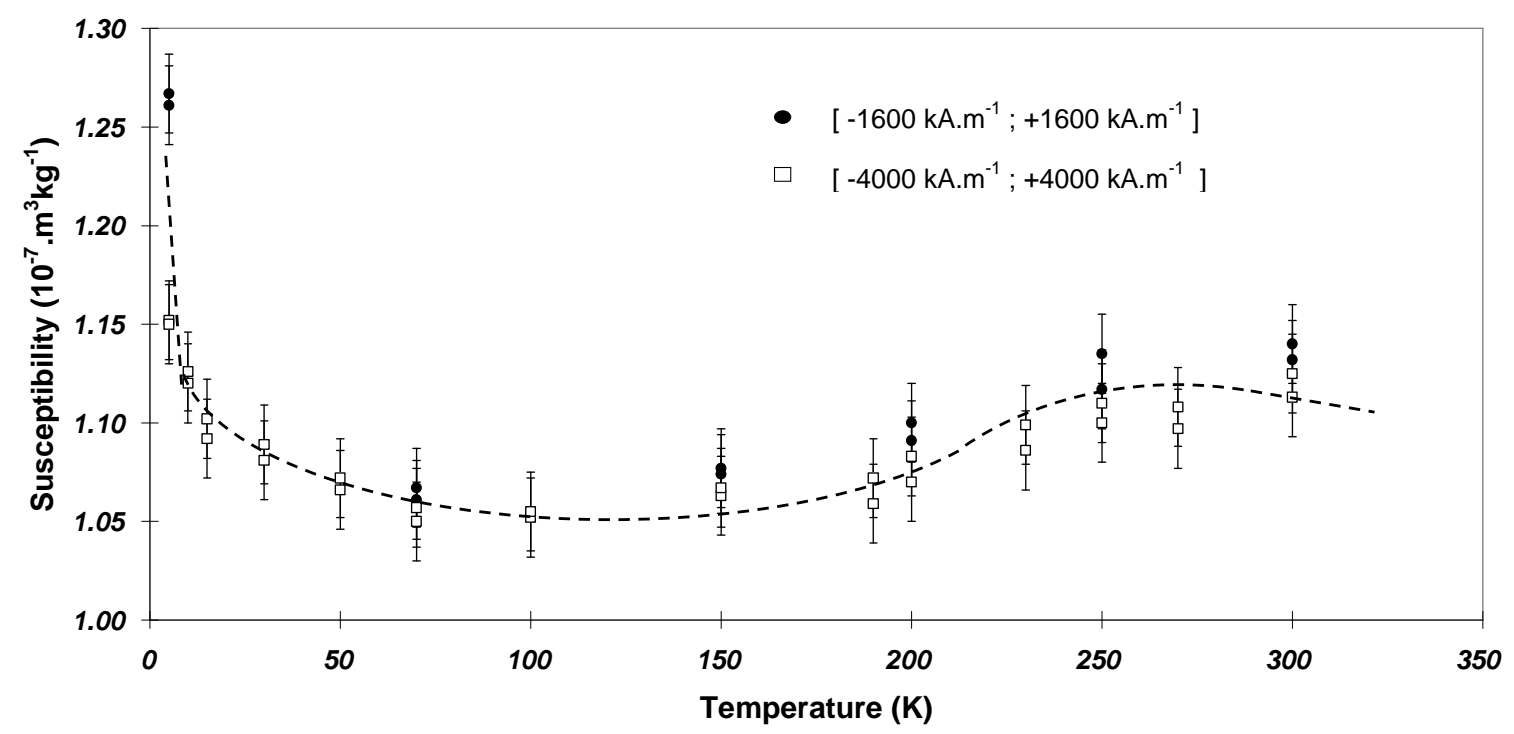

FIG. 3. 


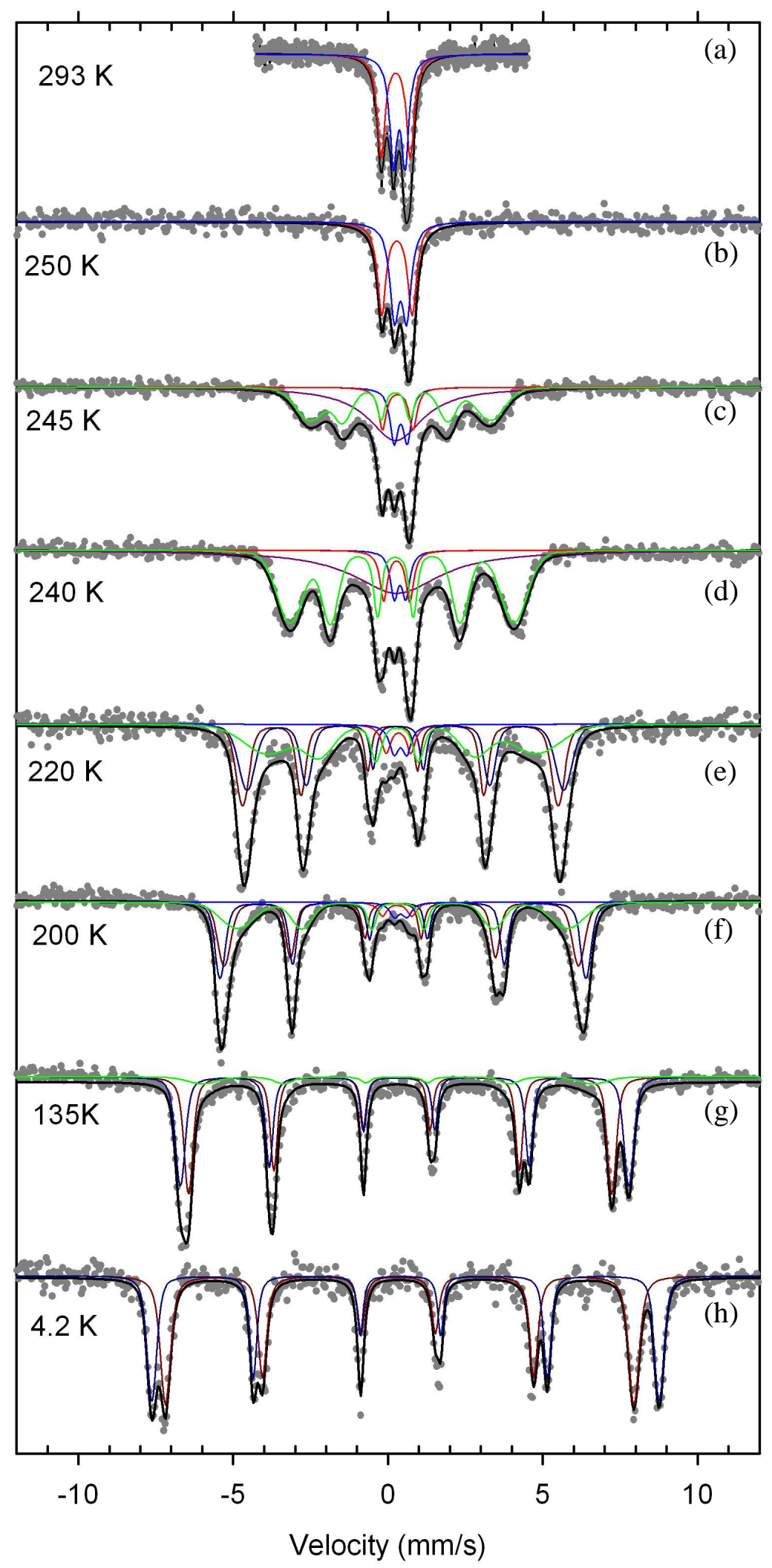

FIG. 4. 


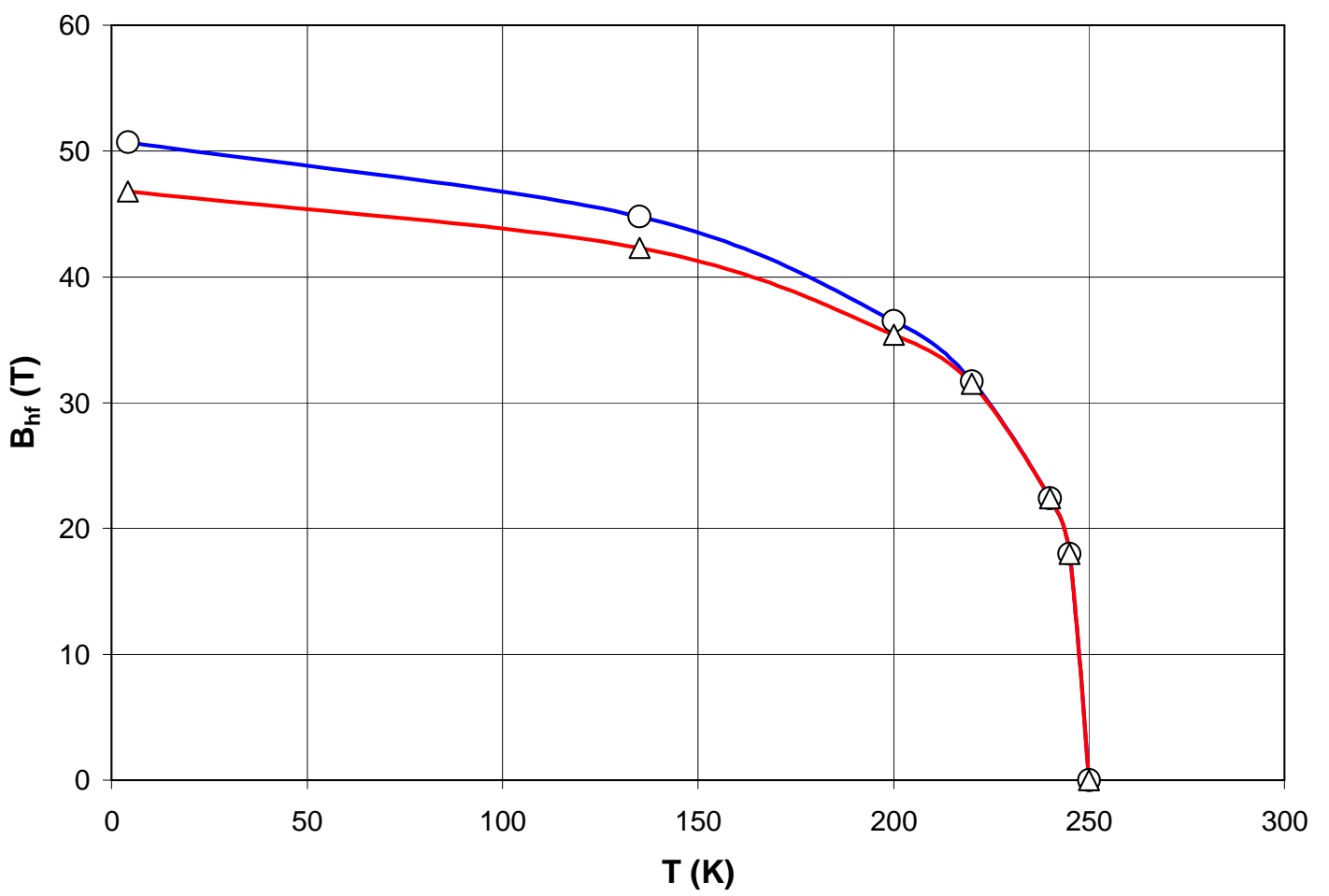

FIG. 5. 


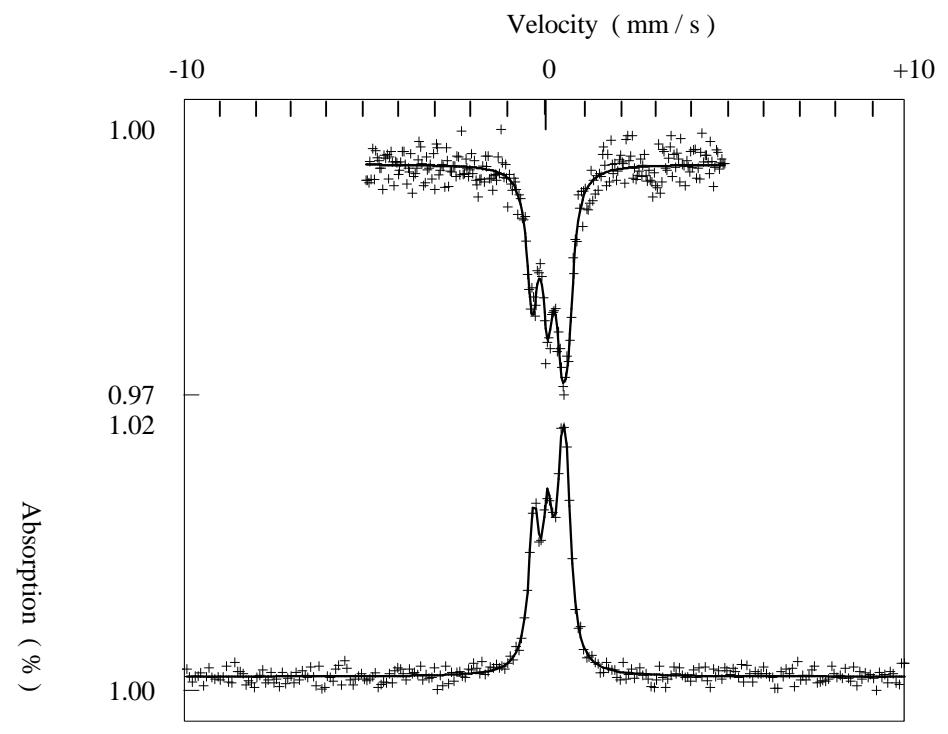

Transmission geometry (TMS)

Reflection geometry (CEMS)

FIG. 6. 
Sub-micron sized $\mathrm{Bi}_{2} \mathrm{Fe}_{4} \mathrm{O}_{9}$ cubes were analyzed by Mössbauer spectrometry.

The transition temperature of the magnetic phase is about $240-250 \mathrm{~K}$.

The surface sites give identical Mössbauer signatures as interior sites. 